\title{
HIGH-ENERGY LATTICE FOR FIRST-BEAM OPERATION OF THE SRF TEST ACCELERATOR AT NML
}

\author{
C. Prokop, ${ }^{1}$ P. Piot,${ }^{1,2}$ and M. Church ${ }^{2}$ \\ ${ }^{1}$ Northern Illinois Center for Accelerator $\&$ \\ Detector Development and Department of Physics, \\ Northern Illinois University, DeKalb IL 60115, USA \\ ${ }^{2}$ Accelerator Physics Center, Fermi National \\ Accelerator Laboratory, Batavia, IL 60510, USA
}

(Dated: September 22, 2011)

\begin{abstract}
The Superconducting Radio Frequency Test Accelerator, a linear electron accelerator currently in construction at Fermilab's New Muon Laboratory, will eventually reach energies of $\sim 900 \mathrm{MeV}$ using four ILC-type superconducting accelerating cryomodules. The accelerator's construction is staged according to cryomodules availability. The first phase that will support first beam operation incorporates one cryomodule. In this Note, we summarize a possible design for the first-beam accelerator configuration.
\end{abstract}




\section{INTRODUCTION}

A superconducting linear electron accelerator currently undergoing construction in FNAL's New Muon Laboratory is planned to eventually reach energies up to $900 \mathrm{MeV}$ using four superconducting ILC-type accelerating modules (cryomodules) [1, 2]. The facility's construction is staged and the initial phase, which will support first beam operation, consists of a $\sim 40 \mathrm{MeV}$ photoinjector followed by one cryomodule (referred to as ACC1) yielding a maximum energy of $\sim 300 \mathrm{MeV}$, and the proposed layout is shown in Fig. 1 . The main goal of this Note is to detail a possible lattice configuration from ACC1's exit to the high-energy (HE) dump located $\sim 98 \mathrm{~m}$ downstream of the cryomodule exit.

The photoinjector will be capable of producing electron beams with variable charges and parameters [3]. The bunch could also be longitudinally compressed using a four-bend magnetic chicane. A telescope composed of four quadrupole magnets downstream of ACC1 allows for matching the beam's Courant-Snyder (C-S) parameters to the desired values at the cryomodule entrance. The ACC1 cryomodule, located $21.9 \mathrm{~m}$ from the photocathode, consists of 8 TESLA-type cavities operating with an average accelerating gradient of $32 \mathrm{MV} / \mathrm{m}$. Finally, a dispersive section starting at $\mathrm{z}=108 \mathrm{~m}$ will enable the measurement of the beam energy and energy spread before being directed to a high-power beam dump (placed at $\mathrm{Z}=133 \mathrm{~m})$.

In subsequent upgrade phases, additional cryomodules will be installed downstream of ACC1, as shown in Fig. 2. Eventually the beamline will also incorporate beam manipulation devices for further bunch compression and phase space repartitioning. In the short term, a simple lattice is desired to transport the beam up to the high-energy (HE) spectrometer beamline.

\section{CRYOMODULE-TO-HE LINE TRANSPORT}

The beamline used to transport the beam between the cryomodule and the HE beamline should be simple, easily reconfigurable (e.g. to accomodate the 2 nd and 3rd cryomodules when they are installed), and able to support the first set of planned experiments. It should also include provisions for beam diagnostics, especially transverse emittance measurements. The design must incorporate a limited number of high-energy (HE) quadrupole magnets. A 


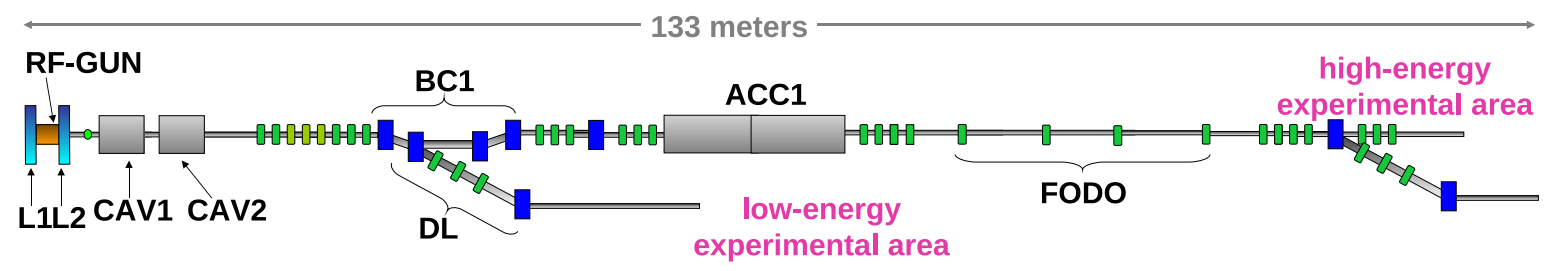

FIG. 1: The "first-beam" design, with only the first cryomodule in place. "RF-Gun" is the photocathode gun, "L1" and "L2" are solenoid magnets, "CAV1" and "CAV2" are nine-cell RF accelerating cavities, "BC1" is the low-energy bunch compressor, "DL" is a dogleg to the low-energy experimental area, "ACC1" is the first cryomodule, and "FODO" is the FODO lattice.

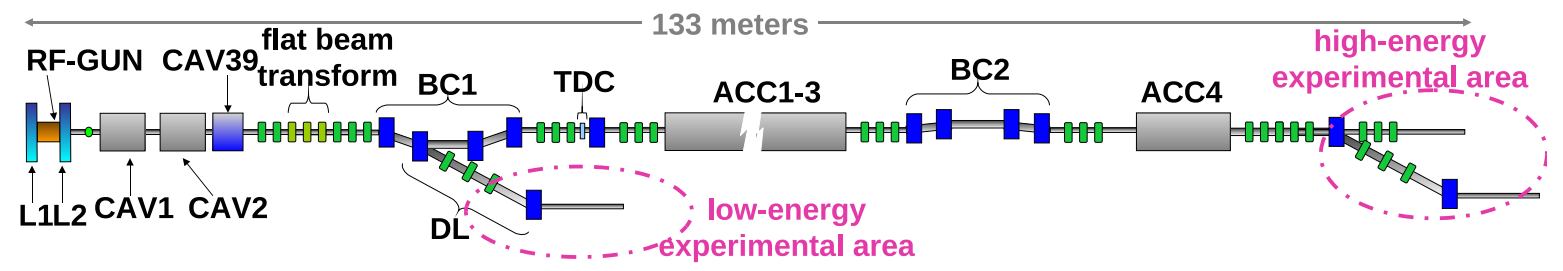

FIG. 2: Longer-term beamline configuration, once four cryomodules (ACC1-ACC4) become available. The beamline will most likely include a second bunch compressor "BC2", a third-harmonic cavity "CAV39", a transverse deflecting cavity "TDC", and three quadrupole magnets to be used for flat-beam transformations.

FODO lattice was chosen as it meets our requirements, can be assembled from the available beamline components, and is easily scalable for reconfigurations (e.g., when other accelerating modules become available).

Between the exit of the first cryomodule and the first dipole of the HE beamline, the lattice incorporates a total of twelve quadrupole magnets: $(i)$ four to match the beam C-S parameters to the FODO lattice, (ii) four to actually comprise the FODO lattice, and (iii) four to match the beam to the desired C-S parameters at the spectrometer entrance. A schematic diagram of the FODO lattice appears in Fig. 3.

Given the available length for the FODO lattice, the small number of allotted quadrupole magnets places onstraints on the betatron function. The minimum and maximum values of the betatron functions in the FODO cell can be parameterized as a function of the betatron 


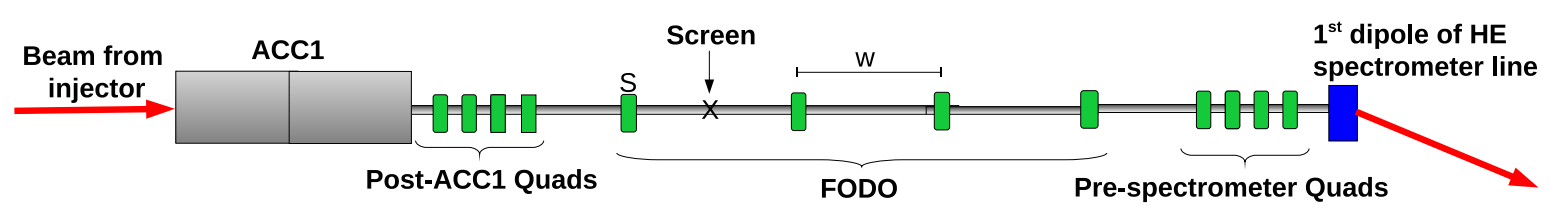

FIG. 3: Schematic of the FODO lattice, showing the four quadrupole magnets after the cryomodule, the four quadrupole magnets that comprise the FODO lattice itself, and the four quadrupole magnets that match into the spectrometer. The "S" and "X" symbols respectively refer to the quadrupole magnet used for the quadrupole magnet scanned and the profile monitor used for the transverse emittance measurements.

phase advance per cell $\Psi$ as [4]

$$
\begin{aligned}
& \beta_{\text {max }}=\frac{w}{\sin \frac{\Psi}{2}} \sqrt{\frac{1+\sin \frac{\Psi}{2}}{1-\sin \frac{\Psi}{2}}} \\
& \beta_{\text {min }}=\frac{w}{\sin \frac{\Psi}{2}} \sqrt{\frac{1-\sin \frac{\Psi}{2}}{1+\sin \frac{\Psi}{2}}}
\end{aligned}
$$

where $w$ is the half-period of the lattice. Tab. I shows the selected values for FODO lattice, and the maximum spot size for several normalized emittances and energies, and Fig. 4 presents $\beta_{\max }$ and $\beta_{\min }$ as functions of $\Psi$ and $w$.

The transverse beam diagnostics place additional upper and lower boundaries on the betatron functions. The total beam size $\sim 8 \sigma$ should fit within the field of view of the imaging system used to measure the beam size. In addition, the beam size should be at least twice as large as the resolution $\sigma_{\text {res }}$ of the beam size measurement method. These two constraints place limits on the lattice functions at the screen $\beta_{\text {screen }}$

$$
\frac{4 \gamma \sigma_{\text {res }}^{2}}{\epsilon_{n}} \leq \beta_{\text {screen }} \leq \frac{d_{F o V} \gamma}{64 \epsilon_{n}}
$$

where $d_{F o V}$ is the field of view of the imaging system, $\sigma_{\text {res }}$ is the resolution of the measurement, $\gamma$ is the beam's relativistic mass factor, and $\epsilon_{n}$ is the transverse normalized emittance; these constraints are shown in Fig. 5.

The C-S parameters entering the cryomodule-to-HE beamline depend on the cryomodule operating parameters as significant ponderomotive focusing occurs in the cryomodule. The transverse ponderomotive force [5] is given by

$$
F_{r}=\frac{\chi_{i} \phi\left(e E_{0}\right)^{2} r}{8 \gamma m_{0} c^{2}} \propto r
$$


TABLE I: Parameters concerning the FODO lattice.

\begin{tabular}{lcccc}
\hline \hline Parameter & Symbol Value Unit \\
\hline Bunch Charge & $\mathrm{Q}$ & 3.2 & $\mathrm{nC}$ \\
Effective Quadrupole Magnet Length & $\mathrm{L}$ & 0.417 & $\mathrm{~m}$ \\
Quadrupole Magnet Separation (center-to-center) & $w$ & 16.4 & $\mathrm{~m}$ \\
Phase Advance & $\Psi$ & 63 & $\mathrm{deg}$ \\
Screen Position from Quadrupole Magnet & $D$ & 8.4 & $\mathrm{~m}$ \\
min betatron function & $\beta_{\min }$ & 20 & $\mathrm{~m}$ \\
max betatron function & $\beta_{\max }$ & 60 & $\mathrm{~m}$ \\
max spot size for $\epsilon_{n}=0.1 \mu \mathrm{m}$ at $300 \mathrm{MeV}$ & $\sigma_{\max }$ & 0.10 & $\mathrm{~mm}$ \\
max spot size for $\epsilon_{n}=0.1 \mu \mathrm{m}$ at $40 \mathrm{MeV}$ & $\sigma_{\max }$ & 0.28 & $\mathrm{~mm}$ \\
max spot size for $\epsilon_{n}=1 \mu \mathrm{m}$ at $300 \mathrm{MeV}$ & $\sigma_{\max }$ & 0.32 & $\mathrm{~mm}$ \\
max spot size for $\epsilon_{n}=1 \mu \mathrm{m}$ at $40 \mathrm{MeV}$ & $\sigma_{\max }$ & 0.88 & $\mathrm{~mm}$ \\
max spot size for $\epsilon_{n}=10 \mu \mathrm{m}$ at $300 \mathrm{MeV}$ & $\sigma_{\max }$ & 1.01 & $\mathrm{~mm}$ \\
max spot size for $\epsilon_{n}=10 \mu \mathrm{m}$ at $40 \mathrm{MeV}$ & $\sigma_{\max }$ & 2.77 & $\mathrm{~mm}$ \\
max spot size for $\epsilon_{n}=50 \mu \mathrm{m}$ at $300 \mathrm{MeV}$ & $\sigma_{\max }$ & 2.26 & $\mathrm{~mm}$ \\
max spot size for $\epsilon_{n}=50 \mu \mathrm{m}$ at $40 \mathrm{MeV}$ & $\sigma_{\max }$ & 6.19 & $\mathrm{~mm}$ \\
\hline \hline
\end{tabular}

where $\gamma m_{0} c^{2}$ is the total particle energy ( $\gamma$ is the lorentz factor $), \phi$ is the particle's phase with respect to the maximum accelerating phase, $r$ is the distance from the cryomodule axis, $e$ is the electric charge, $E_{0}$ is the average accelerating field, and $\chi_{i}$ describes the harmonic content of the spatial field distribution. We take $\chi_{i} \sim 1$ for a standing wave struture which is significant in the first accelerating cavities of the cryomodule as $E_{0} \approx 32 \mathrm{MV} / \mathrm{m}$ and $\gamma m_{0} c^{2} \approx 40 \mathrm{MeV}$. Therefore we should expect the C-S parameter to be significantly affected by the cryomodule parameters, e.g., its operating phase. Though we expect to never operate more than $30^{\circ}$ off-crest, we present more extreme cases in this Note to illustrate the robustness of our design.

A possible solution involves focusing the beam at the cryomodule entrance (i.e. $\alpha>0$ ) and entering the cryomodule with a small beam size $(\beta<20 \mathrm{~m})$. Such a beam would lower the value of $r$ in Eq. 4 and thereby reduce the sensitivity to the ponderomotive force as 

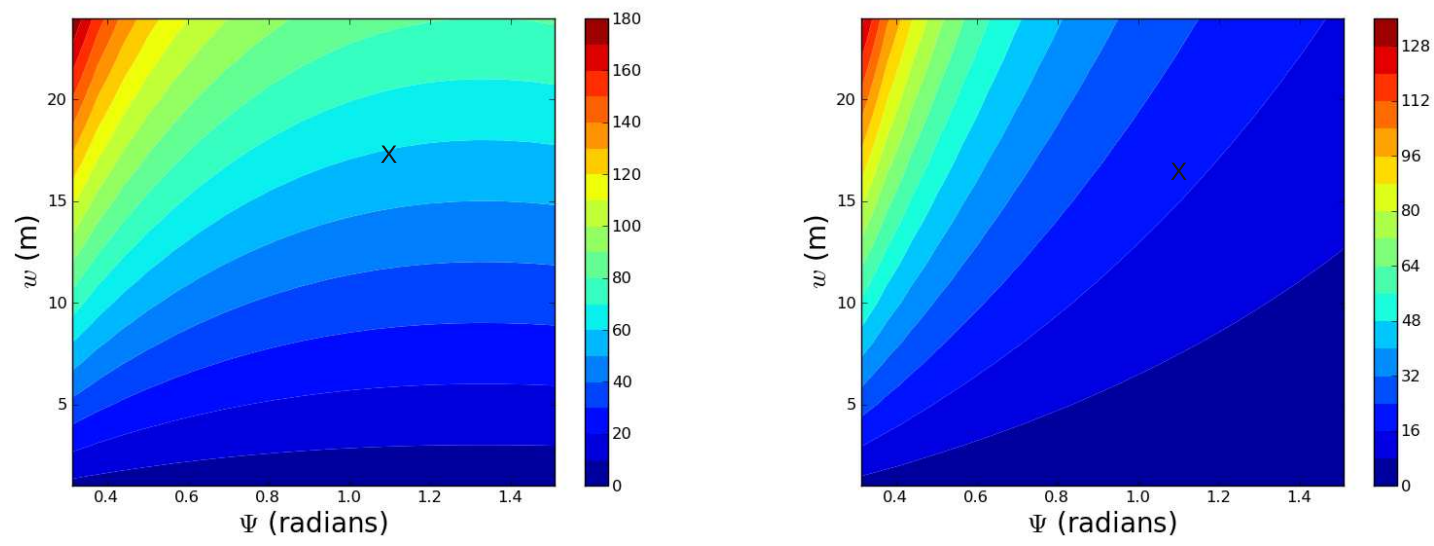

FIG. 4: Maximum (left) and minimum (right) values of the betatron function in the FODO lattice as functions of the betatron phase advance $\Psi$ and the center-to-center distance between quadrupole magnets, $w$. The "X" symbol at $(\Psi, w) \simeq(1.1,17 \mathrm{~m})$ indicates the operating point of the FODO lattice selected for the first beam configuration.

the particles are transported through the cryomodule. A divergent beam will therefore exit the cryomodule. This solution has the overall benefit of reducing the transverse focusing effect of the RF fields, which allows for more consistent behavior across varying peak fields and operating phases. Fig. 6 shows a comparison of the evolution of the betatron function inside the cryomodule for two different initial values of $\beta$. The smaller beam size offers more uniformity of roughly half the phase-dependent variation in $\beta$ and one-third less variation in $\alpha$, which reduces the amount of re-matching that must be done to match into the FODO channel. Transmission through the cryomodule and the C-S parameters at its exit as functions of the C-S parameters at its entrance for various operating phases are shown in Figs. 7-10, using an effective normalized emittance $\epsilon_{n}$ of $100 \mu \mathrm{m}$. The chosen large value for the emittance gives a safe-side estimate and accounts for possible bunch-to-bunch jitter and beam misalignment. 

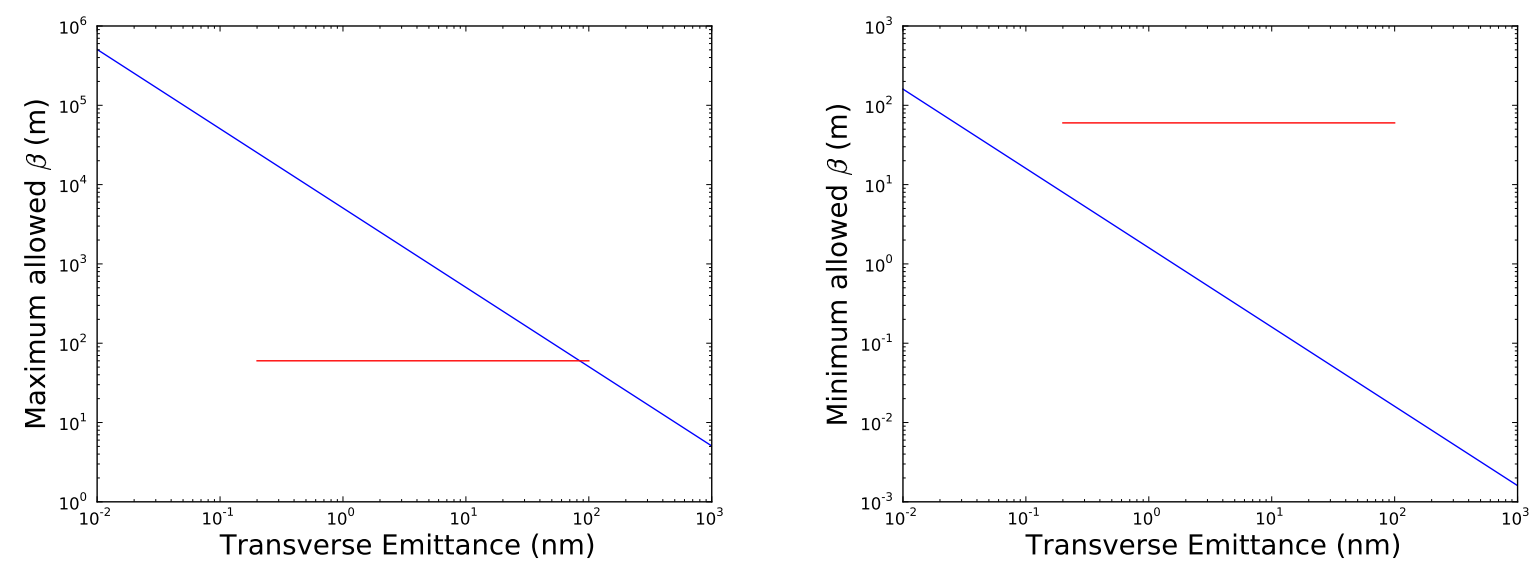

FIG. 5: Maximum (left) and minimum (right) acceptable values for the betatron function (blue traces) at the transverse profile monitor as functions of the transverse geometric (unnormalized) emittance, $\epsilon$. The red line denotes the operating range of the FODO channel specified in Tab. I. The maximum (resp. minimum) value of the betatron function is determined by the size (resp. the resolution) of the transverse profile monitor used for beam size measurement.
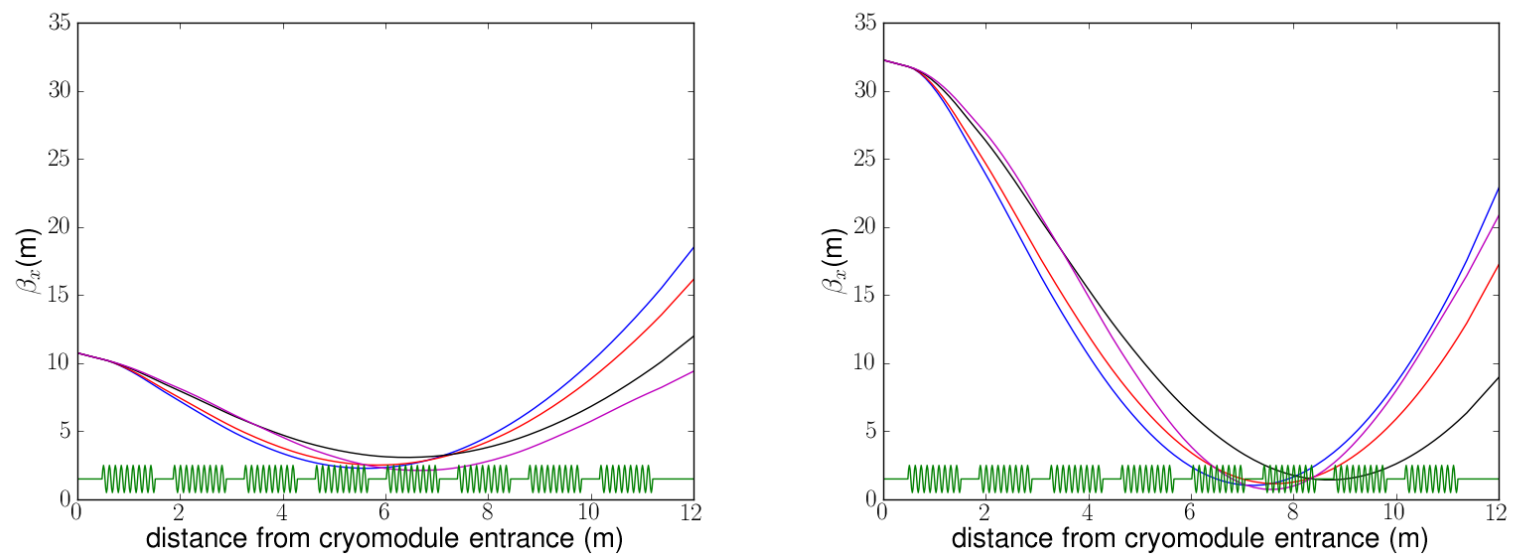

FIG. 6: Lattice functions inside the cryomodule for different operating phases $\left[0^{\circ}\right.$ "on-crest" (red), $30^{\circ}$ (blue), $60^{\circ}$ (black) and $90^{\circ}$ (magenta)] off-crest for $(\alpha, \beta)=(0.5,10.75 \mathrm{~m})$ (left) and $(\alpha, \beta)=$ $(0.5,32.25 \mathrm{~m})$ (right). The green line indicates the position of the eight ILC cavities (each cell shown as a wiggle) composing the cryomodule. A higher initial betatron function leads to beams with larger and more divergent betatron function values downstream of ACC1. The final correlation is $\alpha \in[-2.5,-1.0]$ (left) while $\alpha \in[-4.6,-2.4]$ for the larger initial betatron-function value (right). 

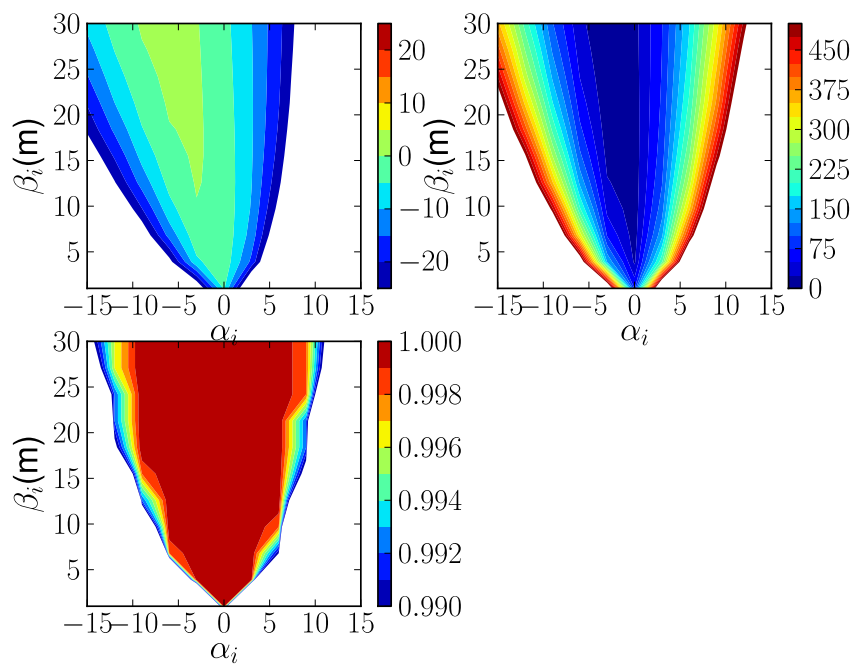

FIG. 7: Contour plots for $\alpha_{f}$ (upper left), $\beta_{f}$ (upper right) and transmission through the cryomodule (lower plot) at the cryomodule exit for the nominal inital C-S parameters $(\alpha, \beta)=(0.5,11 \mathrm{~m})$, with ACC1 operated on-crest $\left(\phi=0^{\circ}\right)$. The units for the $\beta_{f}$, and the transmission contour plots are respectively meters and percents.

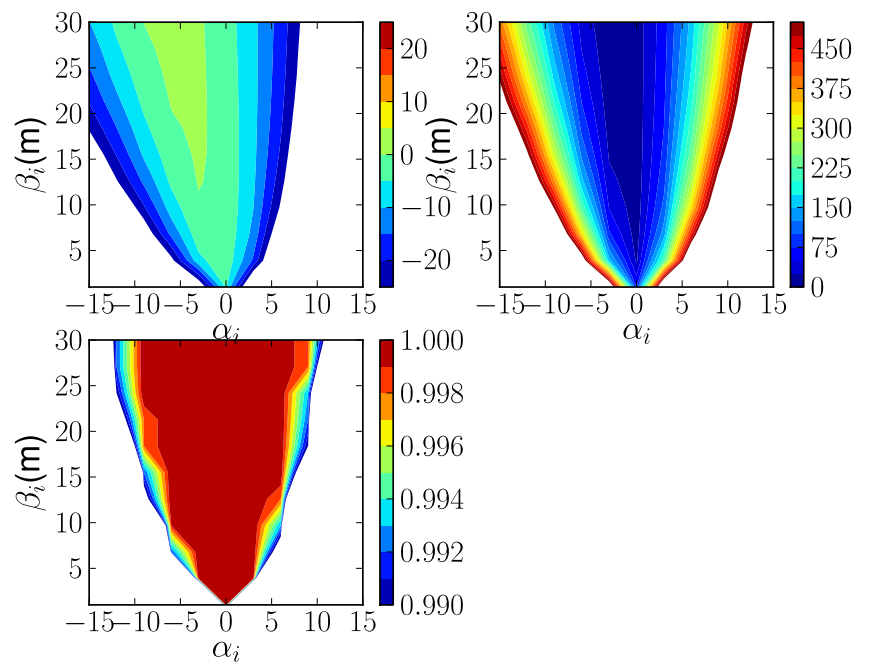

FIG. 8: Contour plots for $\alpha_{f}$ (upper left), $\beta_{f}$ (upper right) and transmission through the cryomodule (lower plot) at the cryomodule exit for the nominal inital C-S parameters $(\alpha, \beta)=(0.5,11 \mathrm{~m})$, with ACC1 operated at $\phi=30^{\circ}$ off-crest. The units for the $\beta_{f}$, and the transmission contour plots are respectively meters and percents. 

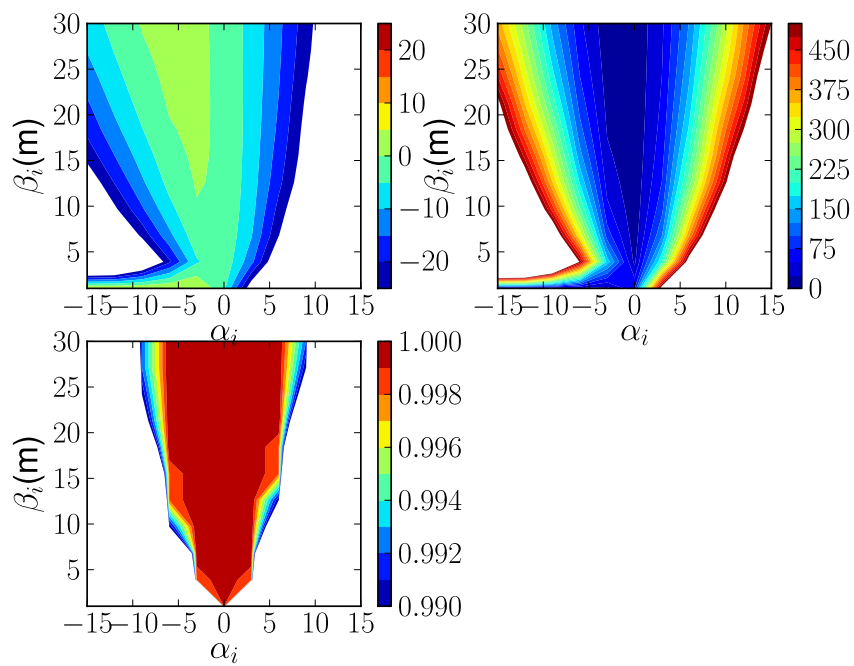

FIG. 9: Contour plots for $\alpha_{f}$ (upper left), $\beta_{f}$ (upper right) and transmission through the cryomodule (lower plot) at the cryomodule exit for the nominal inital C-S parameters $(\alpha, \beta)=(0.5,11 \mathrm{~m})$, with ACC1 operated $\phi=60^{\circ}$ off-crest. The units for the $\beta_{f}$, and the transmission contour plots are respectively meters and percents.

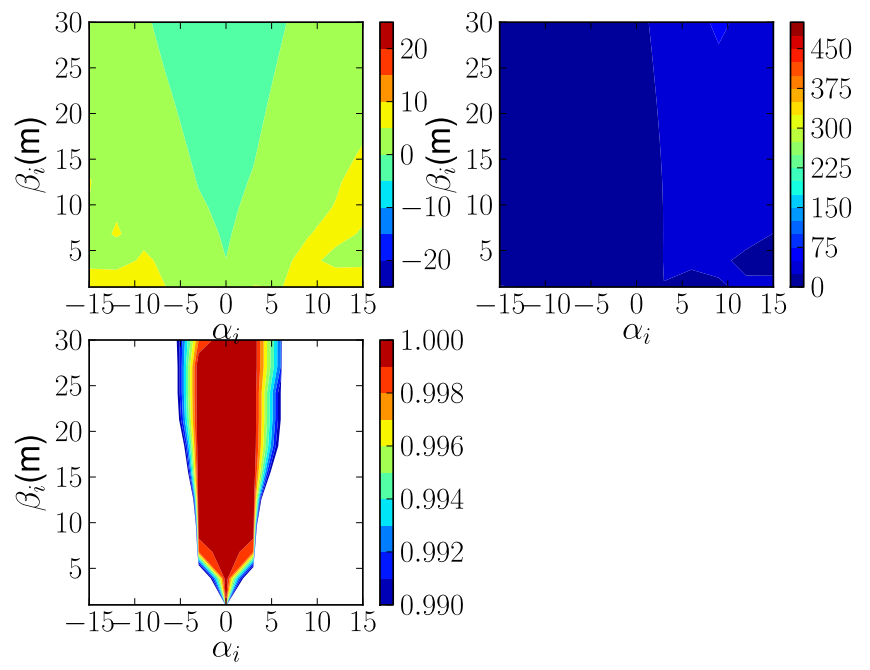

FIG. 10: Contour plots for $\alpha_{f}$ (upper left), $\beta_{f}$ (upper right) and transmission through the cryomodule (lower plot) at the cryomodule exit for the nominal inital C-S parameters $(\alpha, \beta)=(0.5$, $11 \mathrm{~m}$ ), with ACC1 operated $\phi=90^{\circ}$ off-crest. The units for the $\beta_{f}$, and the transmission contour plots are respectively meters and percents. 


\section{HE BEAMLINE}

The HE beamline consists of a dispersive section followed by a beam transport line to the high-power beam dump. The purpose of this dispersive section is to measure the beam energy and energy spread. The dispersive section is composed of a dogleg with two dipoles of \pm 15 deg; shown as D600 and D604 in Fig. 11.

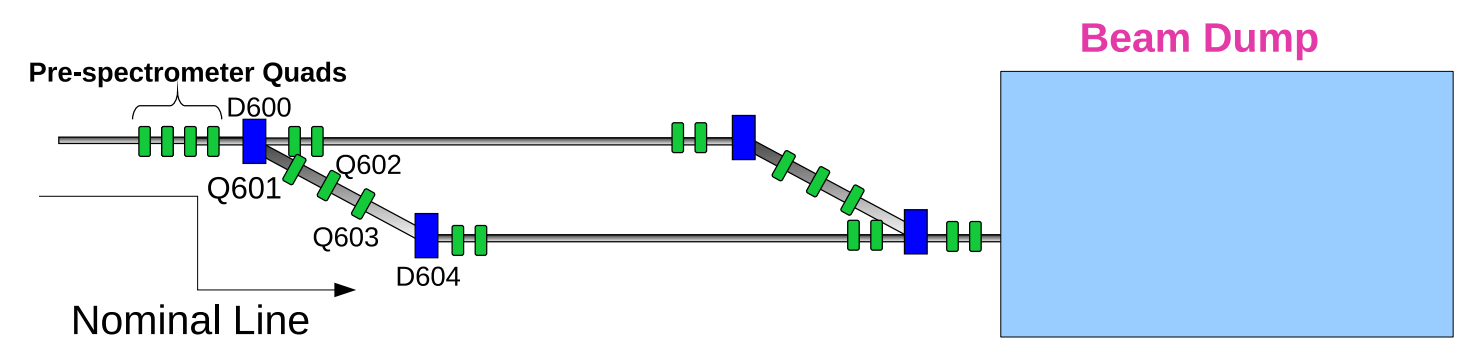

FIG. 11: Schematic diagram of the two spectrometers.
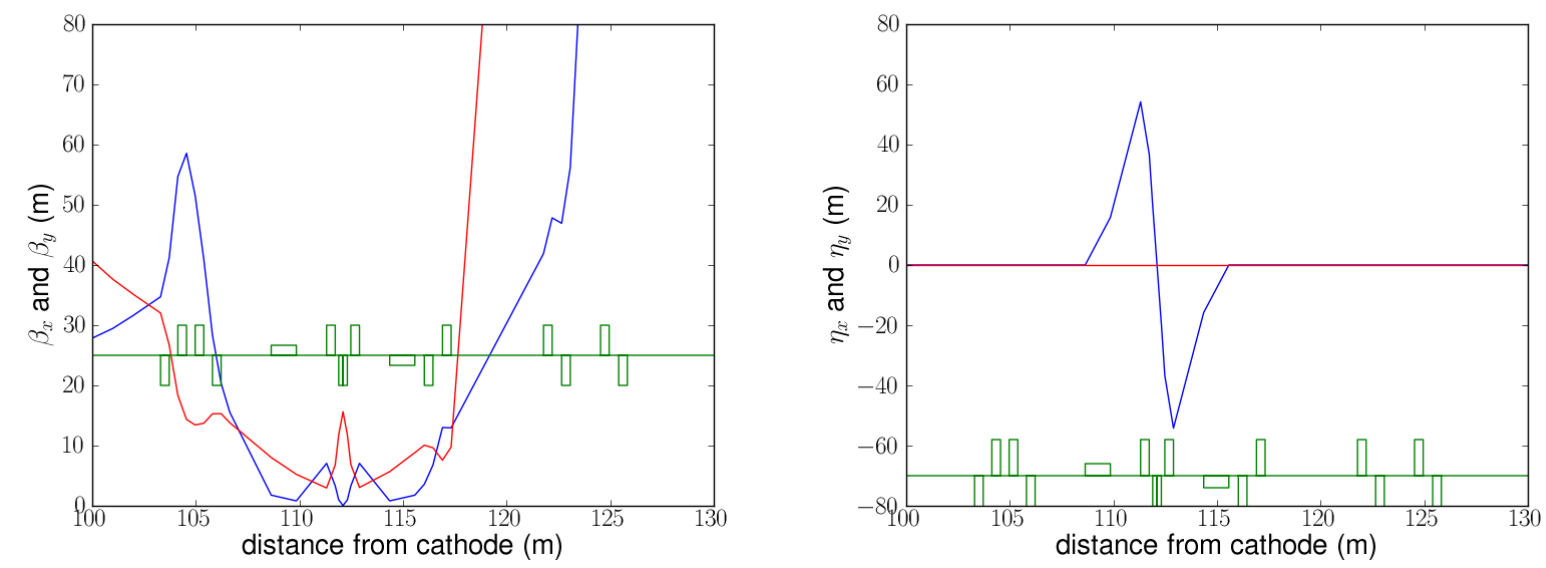

FIG. 12: Evolution of the betatron functions $\beta_{x}$ (red) and $\beta_{y}$ (blue) [Left] and dispersion function $\eta_{x}$ (red) and $\eta_{y}$ (blue) [Right] along the HE spectrometer line.

The dipoles are rectangular dipoles tilted by $7.5 \mathrm{deg}$ to relax the requirement on field uniformity over a smaller transverse region (the beam entrance and exit angles are $+7.5 \mathrm{deg}$ ). Two quadrupole magnets (Q601 and Q603) inserted between the dipoles provide ensure that the horizontal dispersion function and its slope $\left(\eta_{x}, \eta_{x}^{\prime}\right)$ vanish downstream of the second dipole (D604). Such settings provide a dispersion function with two extrema within the dogleg with values of $\pm 0.6 \mathrm{~m}$ (see Fig. 12). A third quadupole (Q602) located at the 
vanishing dispersion point within the dogleg enables further control of the betatron functions. Finally, six quadrupole magnets located downstream of the dogleg are used to defocus the beam on the dump window at $(\mathrm{z}=131 \mathrm{~m})$. In a dispersive section the RMS beam size, at location $s$ is given by

$$
\sigma_{x}=\sqrt{\beta_{x}(s) \epsilon_{x}+\left(\eta_{x}(s) \sigma_{\delta}\right)^{2}} \approx \eta_{x} \sigma_{\delta}
$$

with $\sigma_{\delta}$ is the fractional energy spread (FES). The right-hand-side approximation assumes that $\left(\eta_{x} \sigma_{\delta}\right)^{2} \gg \beta_{x} \epsilon_{x}$. Considering a $5 \mathrm{~cm}$ diameter beampipe and given that $4 \sigma$ of the beam should be contained within the beampipe's aperture, we find a limit of $\sigma_{x} \leq 1.25 \mathrm{~cm}$. The high dispersion point places a limit on the FES of $\sigma_{\delta} \leq 2 \%$ (RMS). As a comparison, a cryomodule with accelerating voltage $V$ operated with an off-crest phase $\phi$ results in a RMS correlated energy spread $\sigma_{\delta_{f}}$

$$
\sigma_{\delta_{f}}=\sqrt{\left(\frac{E_{i}}{E_{i}+e V_{r f} \cos \phi}\right)^{2} \sigma_{\delta i}^{2}+\left(\frac{e V}{E_{i}+e V \cos \phi}\right)^{2}\left(k^{2} \sigma_{z i}^{2}\right) \sin (\phi)^{2}+\frac{3 k^{4}}{4} \sigma_{z i}^{4} \cos (\phi)^{2}}(6)
$$

where $\sigma_{\delta i}$ is the initial FES, $k \equiv 2 \pi / \lambda$ is the wave vector modulus, $\sigma_{z i}$ is the initial RMS bunch length, $E_{i}$ is the initial energy, $e$ is the electron charge, and $\phi$ is the accelerating phase with 0 corresponding to on-crest. Final energy sread is shown as a function of operating phase for various bunch lengths in Fig. 13.

\section{PERFORMANCE STUDIES}

The performance of the designed lattice has been checked for several operating phases of the cryomodules. Two operating scenarios have been explored: One where the upstream telescope is used to rematch the beam into the FODO lattice as the cryomodule phase is varied, and another where the strength of the quadrupoles is adjusted only to account for the varying beam energy. The results shown in Fig. 14-16, demonstrating the ability of the proposed beamline to accommodate ACC1 phase over the full range of anticipated phases (conservatively up to $60^{\circ}$ off-crest, though we expect to never run more than $30^{\circ}$ off-crest). This FODO design allows for intact transport of the beam for a variety of tested cryomodule phases, of at least $30^{\circ}$ off-crest with or without the re-matching of the four quadrupole magnets downstream of the cryomodule exit. The selected spacing of the quadrupole magnets sets minimum and maximum beta functions that can be easily matched for the tested range 


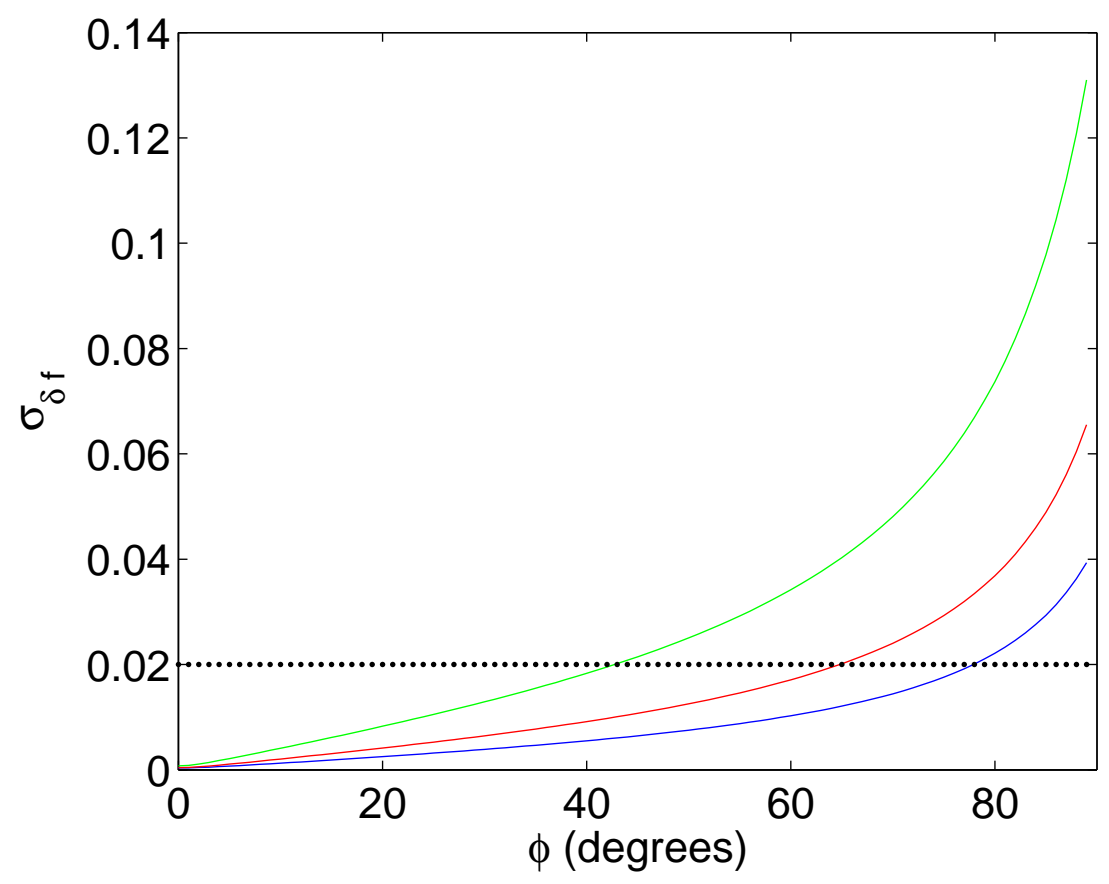

FIG. 13: Final relative fractional energy spread $\sigma_{\delta f}$ as a function of off-crest phase $\phi$, for $\sigma_{z i}=$ 300 (blue), 500 (red) and 1000 (green) $\mu \mathrm{m}$, with $E_{i}=40 \mathrm{MeV}, V_{r f}=210 \mathrm{MV}$, and $\sigma_{\delta i}=0.22 \%$. The black dotted line shows the upper-limit of $2 \%$ that the dispersive section places on our energy spread.

of phases. For optimal behavior, the quadrupoles should be rematched when the phase of the cryomodule is changed to account for the different RF-focusing, but even at $30^{\circ}$ off-crest the deviation from the proper FODO behavior is small (Fig. 15) and the spot size at the beam dump is within $10 \%$ of the on-crest behavior. Since the rematching would require an iterative procedure involving several emittance measurements, it is more appealing from the operational stand point not to have to rematch.

\section{TRANSVERSE EMITTANCE MEASUREMENTS}

Downstream of ACC1, the beam's transverse emittances are foreseen to be measured using the quadrupole magnet scan method [6]: a quadrupole magnet's field will be scanned and the beam size recorded on a downstream optical transition radiation (OTR) viewer or scintillator screen [7]; see Fig. 3. The OTR beam profiling system, to be constructed by RadiaBeam Technologies to Fermilab's specifications, is characterized to have a $14 \mu \mathrm{m}$ and 


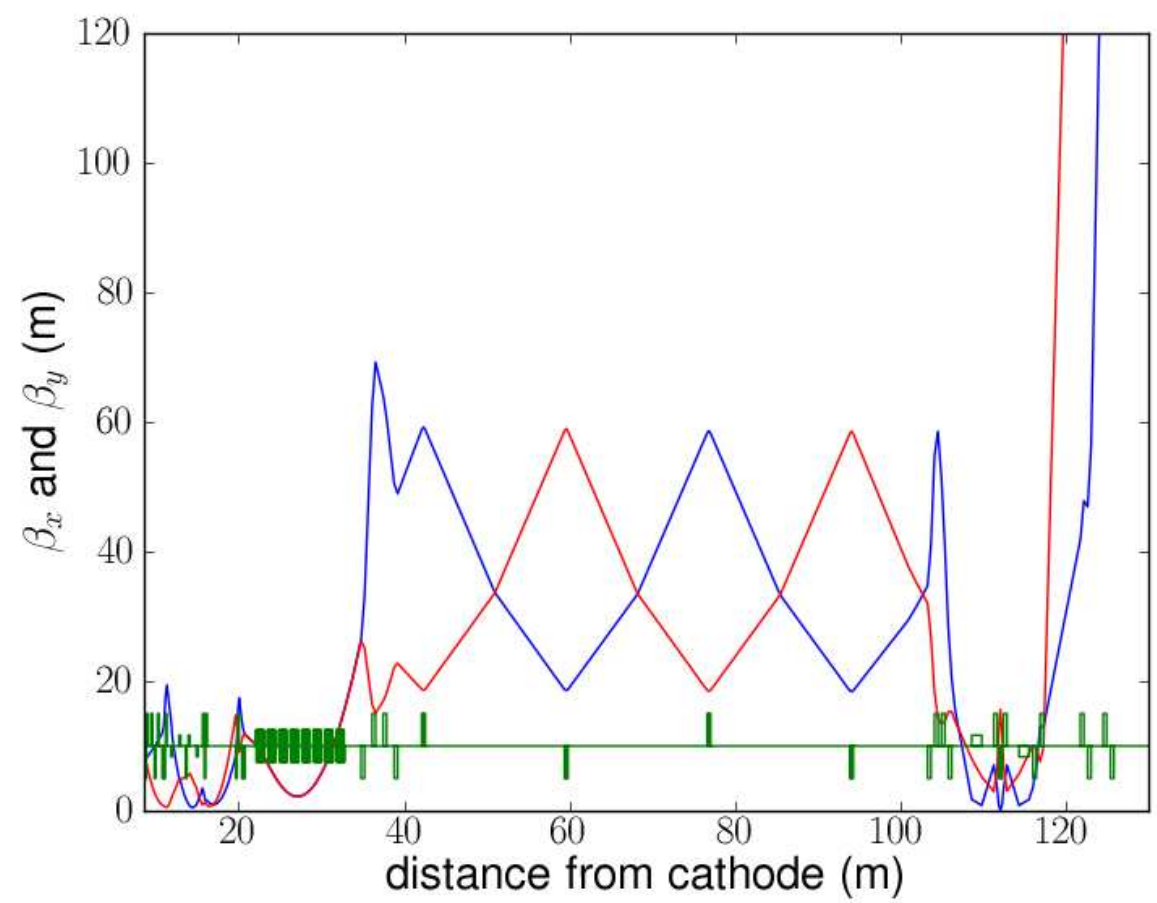

FIG. 14: Betatron functions along the beamline, with ACC1 operated on-crest $\left(\phi=0^{\circ}\right)$.
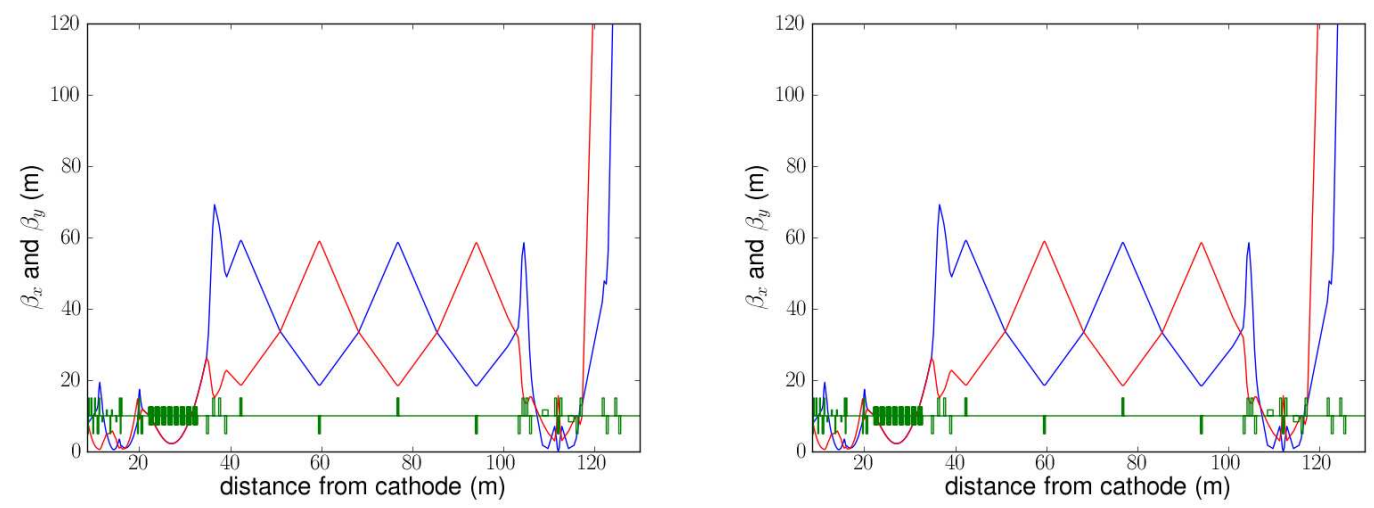

FIG. 15: Betatron functions along the beamline, with ACC1 operated thirty-degrees off-crest $\left(\phi=30^{\circ}\right)$, with (left) and without (right) rematching.

$7 \mu \mathrm{m}$ resolutions for a field of view of 15 and $5 \mathrm{~mm}$ respectively [8], though this design is still tentative. Based on the position of the screen $8.63 \mathrm{~m}$ after the first quadrupole magnet, we use the four quadrupole magnets immediately downstream of the cryomodule to focus to C-S parameters at "S" such that $\frac{\alpha_{x, y}}{\beta_{x, y}}=\frac{1}{D}$ where $D$ is the drift length from the quadrupole magnet's center to the downstream screen. This condition ensures that the beam size on 

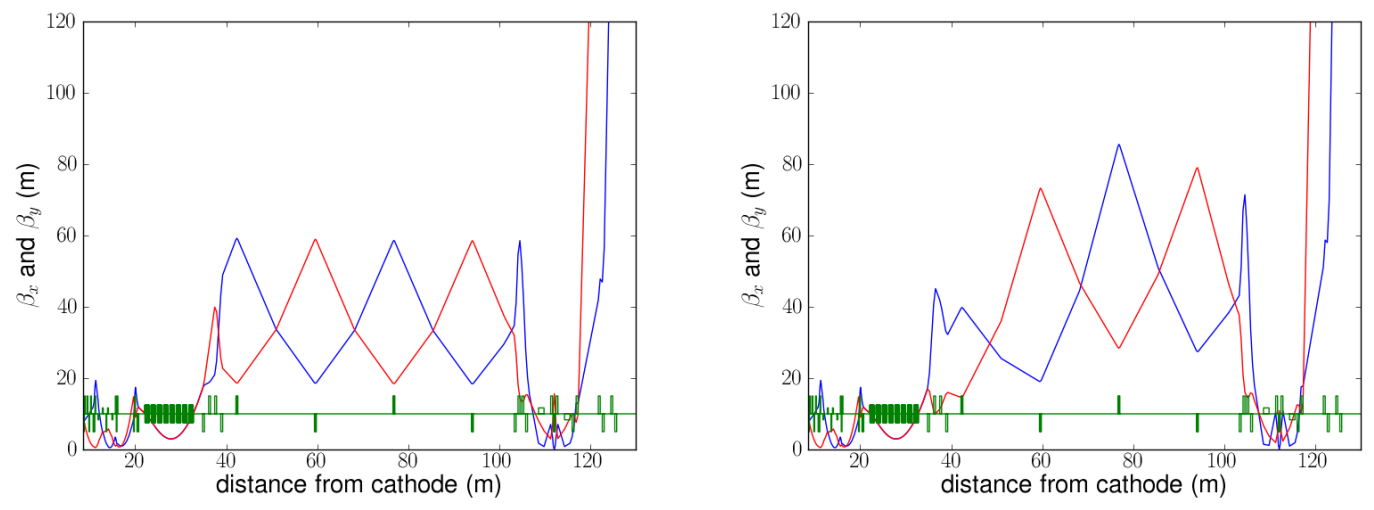

FIG. 16: Betatron functions along the beamline, with ACC1 operated sixty-degrees off-crest $(\phi=$ $60^{\circ}$ ), with (left) and without (right) rematching.

the screen is the smallest at the middle of the scan, i.e. when the quadrupole magnet's field strength $k_{1}$ is set to 0 , and reduces the effect of error.

The quadrupole magnet scan method was simulated in ELEGANT [9] by scanning the strength of the first quadrupole magnet of the FODO lattice and recording the RMS beam size at the location of the profiling system. From the spot size and the strengths of the quadrupole magnets, we are able to recover the beam emittance at the exit of the cryomodule using a least-squares fit method.

To account for resolution effects we, quadratically add the resolution to the simulated beam sizes.

$$
\sigma=\sqrt{\sigma_{\text {sim }}^{2}+\sigma_{\text {res }}^{2}}
$$

We simulate possible statistical error from many potential sources by adding a random fluctuation to the beam size

$$
\sigma_{\text {meas }}=\sigma(1+\Delta \zeta)
$$

where $\Delta$ is the relative amplitude of the statistical uncertainty and $\zeta$ is a random variable following a Gaussian distribution with variance of 1.

As a first case we simply took the nominal beam line settings and scanned the quadrupole magnet's strength. Such a configurtion leads to assymetric evolution of the horizontal and vertical beam sizes; see Fig. 17 (right). On the other hand, when the four preceeding quadrupole magnets are tuned such that the condition $\frac{\alpha}{\beta}=\frac{1}{D}$ is satisfied, one obtains a 
symmetric evolution of the beam sizes which is optimal for achieving similar resolution in the horizontal and vertical emittance measurements.
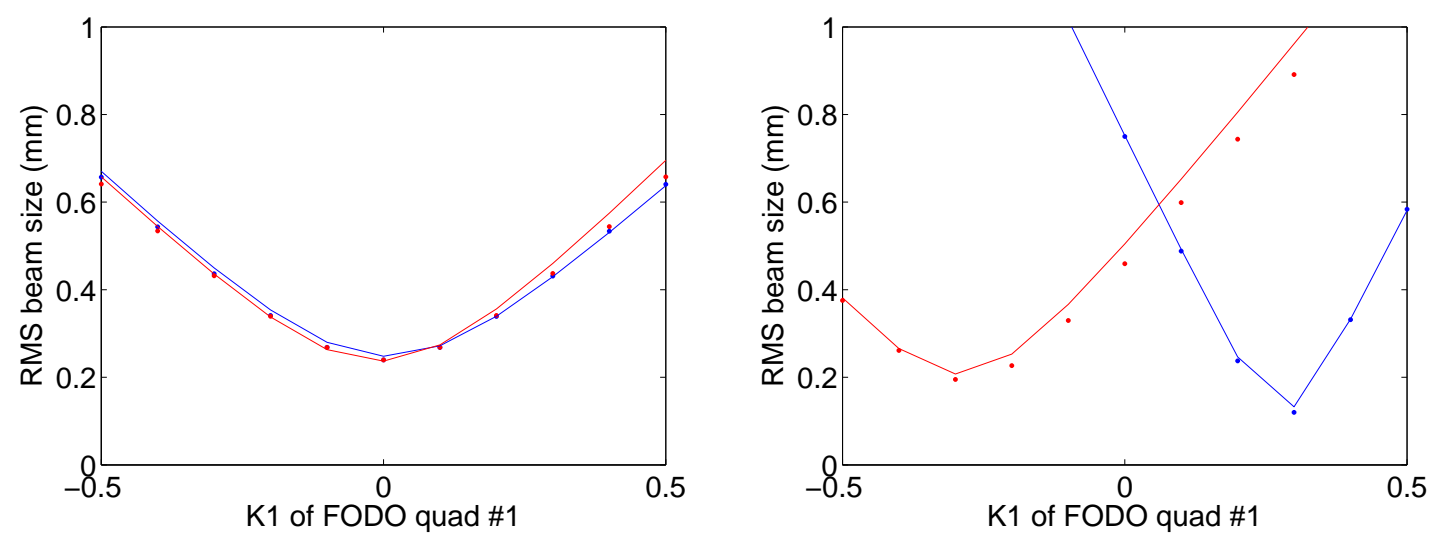

FIG. 17: RMS beam size evolution as a function of scanned quadrupole strength $k_{1}$ with [Left] and without [Right] satisfying the condition $\frac{\alpha_{x y}}{\beta_{x y}}=\frac{1}{D}$ and for simulated $20 \mu \mathrm{m}$ resolution and $10 \%$ of RMS beam jitter/statistical randomness, for a normalized $x$ emittance $\epsilon_{n x}$ of $4.62 \mu \mathrm{m}$ (blue) and a normalized $y$ emittance $\epsilon_{n y}$ of $4.67 \mu \mathrm{m}$ (red). On both plots, the solid lines represent least-squares fits.

Finally, the system

$$
\left(\begin{array}{c}
\sigma_{\text {meas }, 1} \\
\vdots \\
\sigma_{\text {meas }, n}
\end{array}\right)=\left(\begin{array}{ccc}
R_{11,1}^{2} & -2 R_{11,1} R_{12,1} & R_{12,1}^{2} \\
\vdots & \\
R_{11, n}^{2} & -2 R_{11, n} R_{12, n} & R_{12, n}^{2}
\end{array}\right)\left(\begin{array}{c}
\beta_{\text {meas }} \epsilon_{\text {meas }} \\
\alpha_{\text {meas }} \epsilon_{\text {meas }} \\
\gamma_{\text {meas }} \epsilon_{\text {meas }}
\end{array}\right)
$$

is solved by a least-squares fit to recover $\alpha_{\text {meas }}, \beta_{\text {meas }}$ and $\epsilon_{\text {meas }}$, where $R_{11}$ are $R_{12}$ are the $x-x$ and $p_{x}-x$ transfer matrix elements, respectively, which are related to the strengths of the quadrupole magnets used in the scans. The initial parameters $\alpha_{0}, \beta_{0}$ and $\epsilon_{0}$ are compared with the inferred beam from the simulation of the measurements $\alpha_{\text {meas }}, \beta_{\text {meas }}$ and $\epsilon_{\text {meas }}$. The figure of merit $\frac{\epsilon_{\text {meas }}}{\epsilon_{0}}-1$ is plotted in Fig. 18. For these simulations, $\sigma_{\text {res }}=20 \mu \mathrm{m}$. Our calculation follows that derived in Ref. [6] using the uncertainty equal to the resolution, $\Delta \sigma=20 \mu \mathrm{m}$.

For low emittance values, the retrieved emittance is overestimated, and the associated errorbars are large (on the order of 20\%). A histogram of 5000 simulations with random RMS jitter equal to $10 \%$ of measured beam (following the proceedure presented in Ref. [10]) 

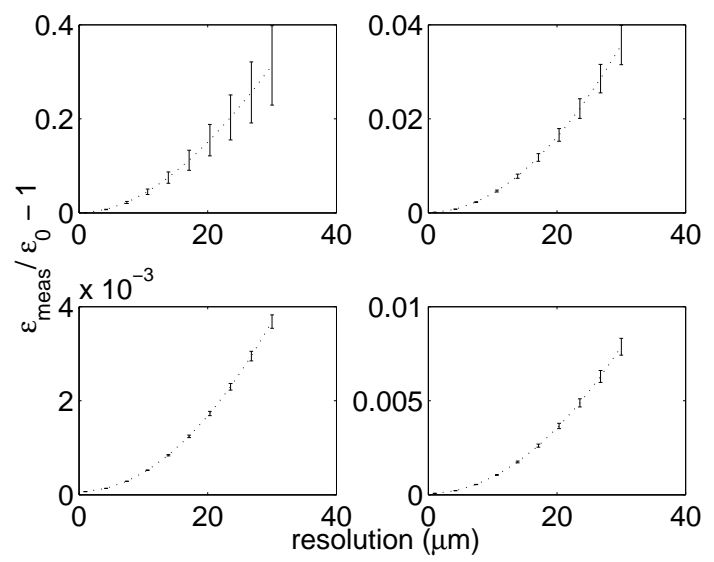

FIG. 18: Relative error on measured emittance $\epsilon_{\text {meas }}$ with respect to initial emittance $\epsilon_{0}$. The value of $\epsilon_{\text {meas }}$ is obtained from a full simulation of the quadrupole magnet field scan technique described in this note. The four plots correspond to initial emittance values of 0.1 (upper left), 1.0 (upper right), 10.0 (lower left), and 4.6 (lower right) $\mu \mathrm{m}$, with a simulated resolution of $\Delta \sigma=20 \mu \mathrm{m}$, without random jitter.

gives an indication of what type of statistical variation we can expect in our measurements (Fig. 19).
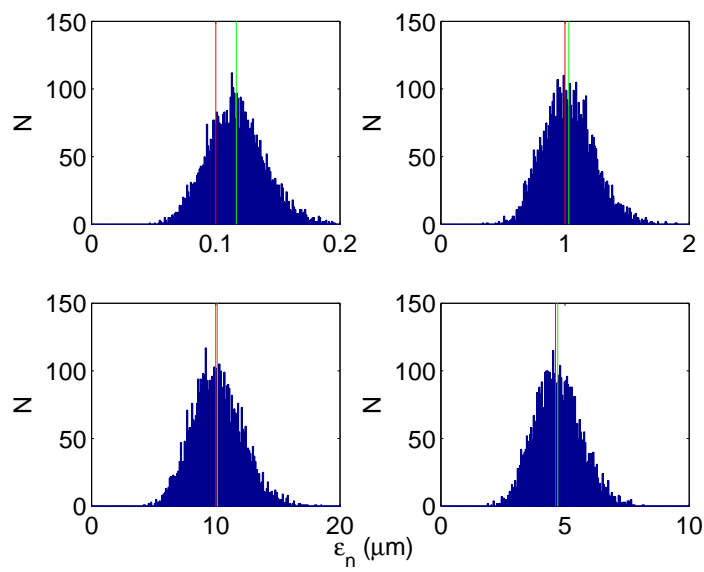

FIG. 19: Histograms of 5000 emittance measurements for 0.1 (upper left), 1.0 (upper right), 10.0 (lower left), and 4.6 (lower right) $\mu \mathrm{m}$ emittances, with a simulated resolution of $\Delta \sigma=20 \mu \mathrm{m}$, and an uncertainty factor $10 \%$ the RMS beam size, showing the statistical deviation that may be expected in the emittance measurements. The red lines are the simulated normalized emittances, while the green lines are the mean of the 5000 measurements. 


\section{CONCLUSION}

In this Note, we presented a possible configuration for the first beam layout of the superconducting RF accelerator test facility. The high-energy beamline includes a FODO lattice and can easily be modified as more accelerating modules become available.

\section{ACKNOWLEDGEMENT}

This work was supported by LANL Laboratory Directed Research and Development (LDRD) program, project 20110067DR and by the U.S. Department of Energy under Contract No. DE-FG02-08ER41532 with Northern Illinois University and under Contract No. DE-AC02-07CH11359 the Fermi Research Alliance, LLC. 
[1] M. Church, S. Nagaitsev, P. Piot, in Proceedings of the 2007 Particle Accelerator Conference (PAC07), June 25-29, 2007, Albuquerque NM, 2942 (2007)

[2] J. Leibfritz, et. al, Proceedings of the 2011 Particle Accelerator Conference (PAC11), March 28-April 1st, 2011, New York, MOP009 (2011)

[3] P. Piot, Y.-E Sun and M. Church, Proceeding of the First International Accelerator Conference (IPAC'10), May 23-28, 2010, Kyoto, Japan, 4313 (2010).

[4] D. Schulte, Linear-Collider School Notes (2010)

[5] J. B. Rosenzweig and L. Serafini, Phys. Rev. E 49, 1599 (1994)

[6] P. Tenenbaum, CLIC-Note-326 (1997)

[7] A. H. Lumpkin, Fermilab Note Beams-doc-2930-v1, posted Nov. 9 (2007)

[8] A. H. Lumpkin, et. al, PAC2011, New York, New York, MOP219 (2011)

[9] M. Borland, "elegant: A Flexible SDDS-compliant Code for Advanced Accelerator Simulation," Advanced Photon Source LS-287, September 2000 (unpublished)

[10] M. Woodley, P. Emma, Proceedings of XX International Linac Conference (LINAC 2000), Monterey, California, MOC19 (2000) 